\title{
Review Article \\ Esophageal Stent for Refractory Variceal Bleeding: A Systemic Review and Meta-Analysis
}

\author{
Xiao-Dong Shao, Xing-Shun Qi, and Xiao-Zhong Guo \\ Department of Gastroenterology, General Hospital of Shenyang Military Area Command, Shenyang 110016, China \\ Correspondence should be addressed to Xiao-Dong Shao; sxdsys608@189.cn
}

Received 18 February 2016; Revised 15 June 2016; Accepted 20 June 2016

Academic Editor: Michel Kahaleh

Copyright ( 2016 Xiao-Dong Shao et al. This is an open access article distributed under the Creative Commons Attribution License, which permits unrestricted use, distribution, and reproduction in any medium, provided the original work is properly cited.

Background. Preliminary studies suggest that covered self-expandable metal stents may be helpful in controlling esophageal variceal bleeding. Aims. To evaluate the effectiveness and safety of esophageal stent in refractory variceal bleeding in a systematic review and meta-analysis. Methods. A comprehensive literature search was conducted on PubMed, EMBASE, and Cochrane Library covering the period from January 1970 to December 2015. Data were selected and abstracted from eligible studies and were pooled using a random-effects model. Heterogeneity was assessed using $I^{2}$ test. Results. Five studies involving 80 patients were included in the analysis. The age of patients ranged from 18 to 91 years. The mean duration of follow-up was $46.8 \mathrm{~d}$ (range, 30-60 d). The success rate of stent deployment was $96.7 \%$ (95\% CI: 91.6\%-99.5\%) and complete response to esophageal stenting was in $93.9 \%$ (95\% CI: $82.2 \%-99.6 \%$ ). The incidence of rebleeding was $13.2 \%$ (95\% CI: $1.8 \%-32.8 \%$ ) and the overall mortality was $34.5 \%$ (95\% CI: $24.8 \%-$ $44.8 \%)$. Most of patients (87.4\%) died from hepatic or multiple organ failure, and only $12.6 \%$ of patients died from uncontrolled bleeding. There was no stent-related complication reported and the incidence of stent migration was $21.6 \%$ (95\% CI: $4.7 \%-46.1 \%)$. Conclusion. Esophageal stent may be considered in patients with variceal bleeding refractory to conventional therapy.

\section{Introduction}

Esophageal varices are portosystemic collateral venous channels related to portal hypertension and present in nearly $50 \%$ of patients diagnosed with cirrhosis [1]. They initially develop as small varices that gradually dilate at a rate of $5 \%$ per year. Acute variceal bleeding is a severe complication of portal hypertension causing $70 \%$ of all upper gastrointestinal bleeding episodes in patients with portal hypertension [2]. With the use of current prophylactic therapies of variceal bleeding, including nonselective beta-blockers and band ligation, the rate of first variceal bleeding is about $8 \%$ per year [3]. Risk factors of variceal bleeding mainly include the severity of liver disease, the size of varix, and the presence of red wale marks [4]. Hemodynamic studies suggest a close association of hepatic venous pressure gradient with the risk of variceal bleeding [5]. Prognostic factors for death include the severity of variceal bleeding, the degree of hepatic dysfunction, and the development of complications including acute renal failure and bacterial infections [6]. Mortality of patients with variceal bleeding has decreased significantly over the last two decades with the implementation of intensive care management, including the use of antibiotic prophylaxis and endoscopic variceal band ligation $[7,8]$. However, the treatment of refractory bleeding and prevention of early rebleeding are still a challenge for physicians [9].

The aim of treatment of acute variceal bleeding is to correct hypovolemia, prevent complications, and achieve hemostasis. After resuscitation, airway protection, and prevention of complication, the initial approach for variceal bleeding is a combination of vasoactive drugs, antibiotics, and endoscopic therapy [10]. About $80 \%-90 \%$ of acute variceal bleeding episodes are successfully controlled by endoscopic therapy [11]. In $10 \%-20 \%$ of patients acute variceal bleeding is not controlled with this primary endoscopic and pharmacological therapy, which is known as refractory variceal bleeding [12]. More aggressive therapies may be used to deal with refractory variceal bleeding. Early TIPS should be considered in patients at high risk of treatment failure after initial endoscopic and pharmacological therapy [13]. 
Although rescue TIPS is very effective in controlling the bleeding, the mortality is high (25-60\%) due to the poor condition of patients [14]. Balloon tamponade aims at achieving hemostasis by direct compression of the bleeding varices. However, after deflating the balloon, the recurrence rate of bleeding is about $50 \%$ [15]. Complications can occur in more than $25 \%$ of the patients treated with balloon tamponade with fatal ones in $5 \%$ of the cases. So it is recommended that balloon tamponade should only be used by skilled and experienced personnel in intensive care facilities [16]. Additionally, the use of the tube is highly unpleasant for patients. Surgical procedures are employed less frequently with the development of endoscopic therapy and TIPS. Although surgical procedures can effectively control variceal bleeding, the mortality remains high (45-75\%) and hepatic encephalopathy is a major complication after shunt procedures [17]. The above-mentioned limitations prompt physicians to seek other modalities to deal with refractory esophageal variceal bleeding.

Self-expandable metal stents (SEMSs) are mainly used in various benign and malignant esophageal diseases, such as stricture, tracheoesophageal fistula, perforation, and achalasia [18]. Anecdotal experience suggests that the covered SEMSs may be useful in controlling esophageal variceal bleeding [19-25]. Since refractory variceal bleeding is an uncommon complication of cirrhosis, most of studies on the effectiveness of esophageal stent have been limited to a small number of patients [26-32]. A recent randomized controlled trial included only 13 patients undergoing esophageal stenting for refractory variceal bleeding [33]. The purpose of this study was to evaluate the effectiveness and safety of esophageal stent in patients with refractory variceal bleeding by pooling all available evidence in a systematic review with meta-analysis.

\section{Materials and Methods}

2.1. Literature Search. A comprehensive literature search was conducted using PubMed, EMBASE, and Cochrane Library for the period from January 1970 to December 2015. The search terms included, in different combinations, "esophageal stent", "self-expandable metal stents", "variceal bleeding", "variceal hemorrhage", and "endoscopic hemostasis". The search was limited to studies in humans published in English. References of eligible articles and review articles were manually searched.

2.2. Selection of Articles. The selection criteria were studies in (1) patients with cirrhosis irrespective of etiology; (2) patients with refractory variceal bleeding; and (3) series that included at least 10 patients. Case reports or series with fewer than 10 patients were excluded. After excluding duplicate articles, article titles and abstracts were screened by a reviewer (SXD). Each eligible article was reviewed in full text.

2.3. Data Extraction. Data were abstracted by the same reviewer and entered into an Excel spreadsheet (Microsoft Corp., Redmond, Washington). The following information was abstracted from each study: author, country, publication year, publication type, study design, participants, and outcome of interest (success rate of stent deployment, response to esophageal stent, rebleeding rate, overall mortality, cause of death, stent-related complications, and incidence of stent migration).

\subsection{Definitions}

Refractory Variceal Bleeding. Patients with active variceal bleeding were unresponsive to pharmacologic and endoscopic therapy and required transfusion.

Response to Esophageal Stent. Clinically, response was considered as complete hemostasis if the patients' symptoms of bleeding resolved. No response was defined as persistent or worsening symptoms of bleeding. Endoscopically, response was defined as no active bleeding or oozing from a varix on endoscopy. No response was defined as active bleeding or oozing found on endoscopy. Eligible studies used endoscopic and/or clinical criteria to assess the response to esophageal stent.

Esophageal Stent-Related Complications. Esophageal stentrelated complications include esophageal tear or perforation, pulmonary dysfunction, aspiration pneumonia, asphyxia, and esophageal ulcer leading to bleeding which need further specific treatment.

Stent Migration. Esophageal stent migration was defined as stent migrating proximally or distally from the place where stents were deployed initially. Radiological examinations were necessary.

Overall Mortality. Overall mortality was defined as any death events throughout the follow-up period. The followup period varied across the studies, with the longest duration being $60 \mathrm{~d}$.

2.5. Statistical Analysis. Data from eligible studies were pooled using a random-effects model with StatsDirect statistical software Version 2.7.8. Outcomes are expressed as proportions (percentages) with $95 \%$ CIs. The pooled analyses are presented as forest plots. Statistical heterogeneity between studies was assessed using the Cochran $Q$ test and $I^{2}$ statistic. $I^{2}$ value of greater than $50 \%$ or a $P$ value of less than 0.05 for the $Q$ statistic was taken to indicate significant heterogeneity.

\section{Results}

3.1. Literature Search Results. Five studies involving a total of 80 patients were included in the analyses. Eight studies were excluded because each had a small number of study subjects. Figure 1 summarizes the results of the literature search. Table 1 summarizes the characteristics of the 5 eligible studies.

3.2. Characteristics of Study Participants. Seventy-one patients were male. The age ranged from 18 to 91 years. The mean duration of follow-up was $46.8 \mathrm{~d}$ (range, 30-60 d). 


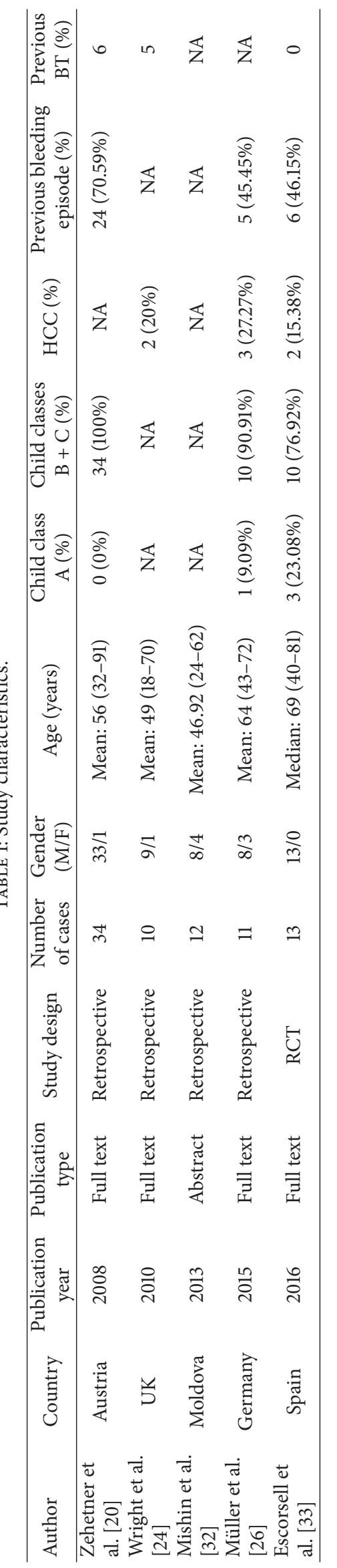




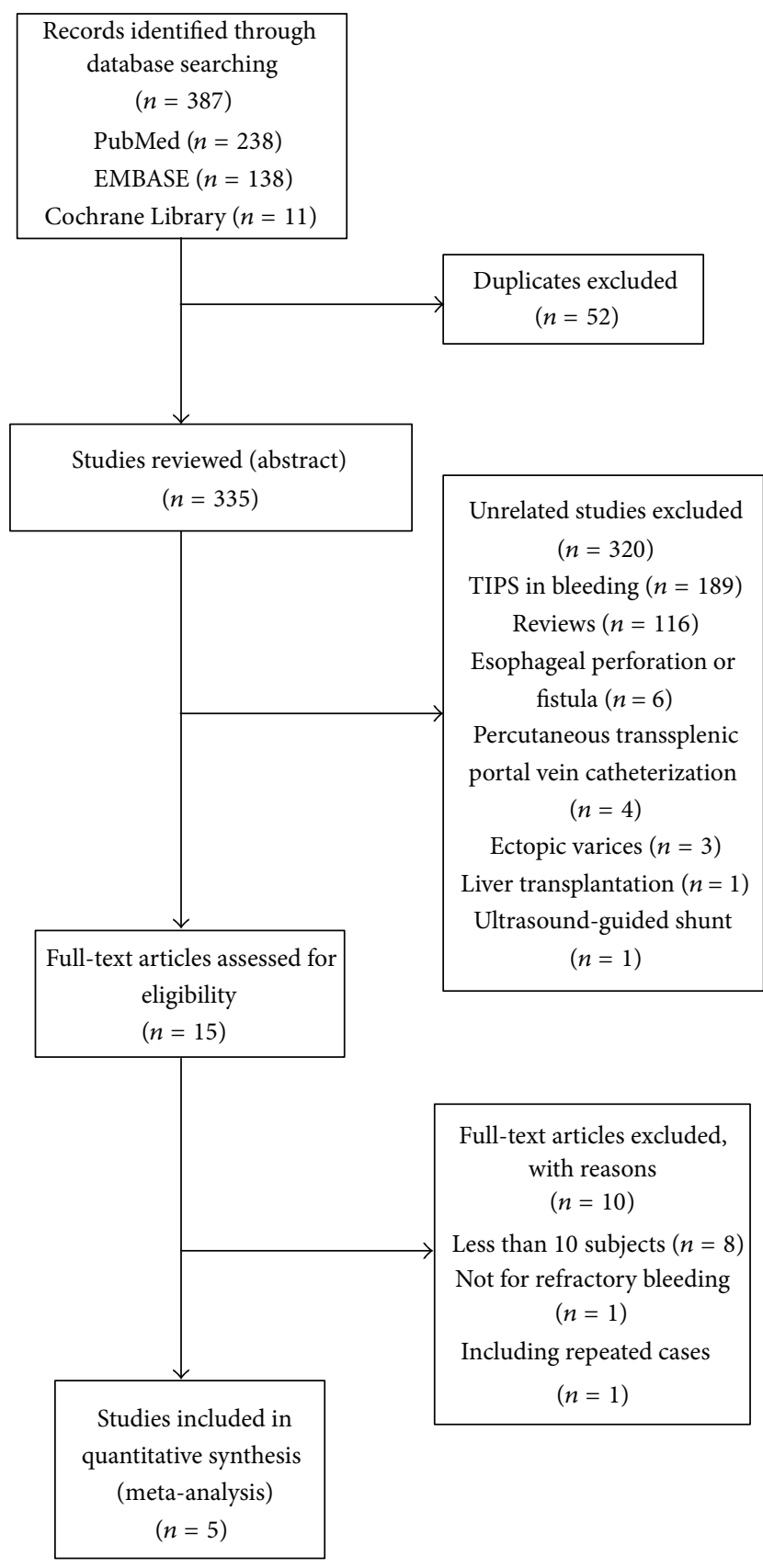

FIgURE 1: Study selection flow chart. Of a total of 387 studies, only 5 studies met selection criteria. TIPS indicates transjugular intrahepatic portosystemic shunt.

Table 2 shows the results of the various outcomes of the individual studies.

3.3. Stent Deployment. The success rate of stent deployment was $96.7 \%$ (95\% CI: 91.6\%-99.5\%) (Figure 2). Heterogeneity was not significant among the studies $\left(I^{2}=6.8 \% ; P=0.37\right)$.

3.4. Response to Esophageal Stent. Complete response to esophageal stenting was in 93.9\% (95\% CI: 82.2\%-99.6\%)

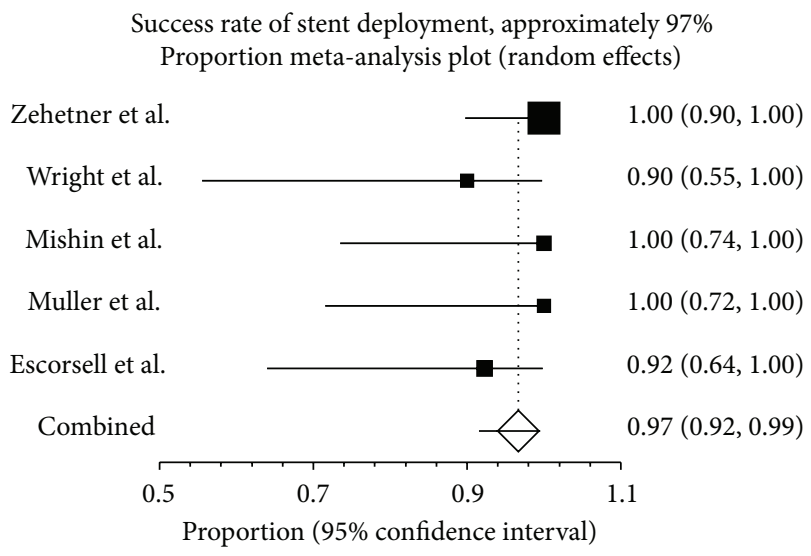

FIGURE 2: Deployment of esophageal stent in patients with refractory variceal bleeding. The esophageal stents were successfully deployed in $96.7 \%$ (95\% CI: $91.6 \%-99.5 \%$ ) of the 80 patients in the 5 studies. There was no heterogeneity among the studies $(P=0.37)$.

(Figure 3(a)). Heterogeneity was significant among the studies $\left(I^{2}=62.5 \% ; P=0.03\right)$. The incidence of rebleeding was 13.2\% (95\% CI: 1.8\%-32.8\%) (Figure 3(b)). Heterogeneity was significant among the studies $\left(I^{2}=78.1 \% ; P=0.00\right)$.

3.5. Mortality. The overall mortality was $34.5 \%$ (95\% CI: 24.8\%-44.8\%) (Figure 4(a)). Heterogeneity was not significant among the studies $\left(I^{2}=0 ; P=0.60\right) .87 .4 \%$ (95\% CI: $71.2 \%-97.5 \%)$ of patients died from hepatic or multiple organ failure (Figure 4(b)). Only 12.6\% (95\% CI: $2.5 \%-28.8 \%$ ) of patients died from uncontrolled bleeding (Figure $4(\mathrm{c})$ ).

3.6. Complications. There was no stent-related complication reported.

3.7. Stent Migration. The incidence of stent migration was 21.6\% (95\% CI: 4.7\%-46.1\%) (Figure 5). Heterogeneity was significant among the studies $\left(I^{2}=81.6 \% ; P=0.00\right)$.

\section{Discussion}

This study shows the following: (1) esophageal stent was successfully deployed in $96.7 \%$ of patients with refractory variceal bleeding; (2) after successful deployment, the hemostasis rate was $93.9 \%$; (3) the rate of rebleeding after esophageal stent is $13.2 \%$; (4) no stent-related complications were reported in the 5 studies; (5) the overall mortality of patients was $34.5 \%$; (6) a majority of patients with refractory variceal bleeding died of hepatic or multiple organ failure and only a minority of patients died from uncontrolled bleeding.

Variceal bleeding is a lethal complication of liver cirrhosis. When acute variceal bleeding fails to respond to pharmacological or endoscopic treatment, balloon tamponade is often undertaken $[34,35]$. Although balloon tamponade is effective in controlling bleeding, it can be associated with complications such as perforation, asphyxia, and aspiration pneumonia [36-40]. And when balloon is extracted, the 


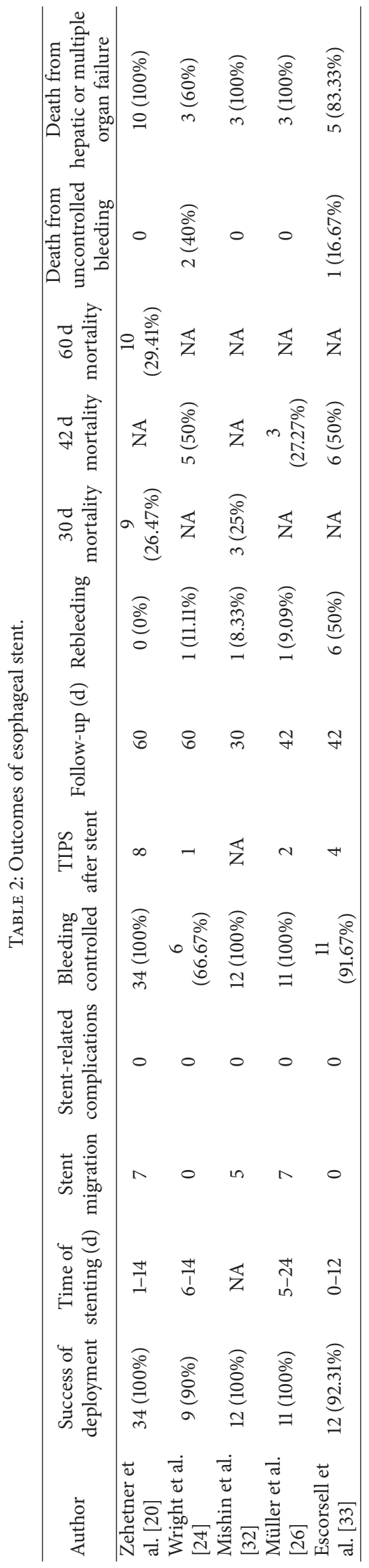




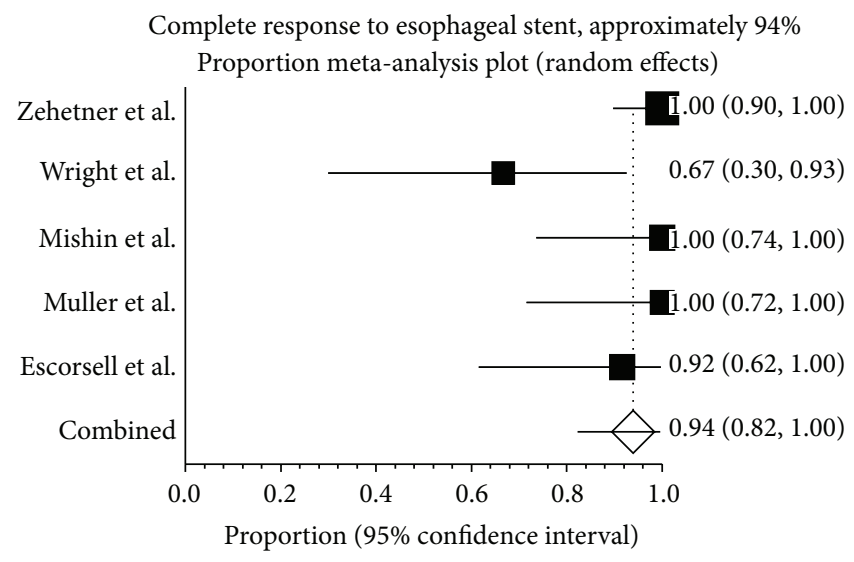

(a)

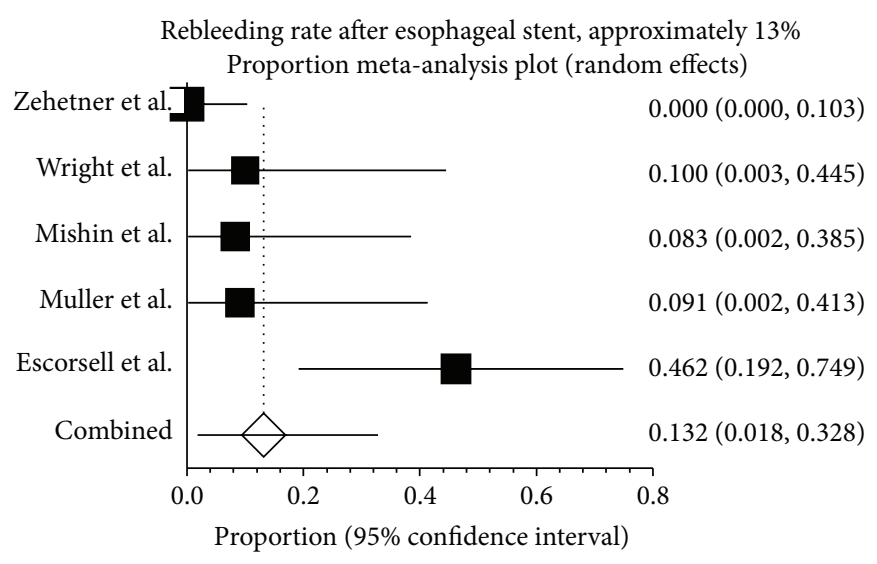

(b)

FIGURE 3: Response to esophageal stent in refractory variceal bleeding. (a) Forest plot shows that $93.9 \%$ (95\% CI: $82.2 \%-99.6 \%$ ) of the 80 patients in the 5 studies had a complete response (resolution of acute variceal bleeding without further need for other treatments) after deployment of esophageal stent. There was evidence of heterogeneity among the studies $(P=0.03)$. (b) Just over one-tenth (13.2\%) of the patients treated with esophageal stents rebled after this procedure. There was evidence of heterogeneity among studies $(P=0.00)$.

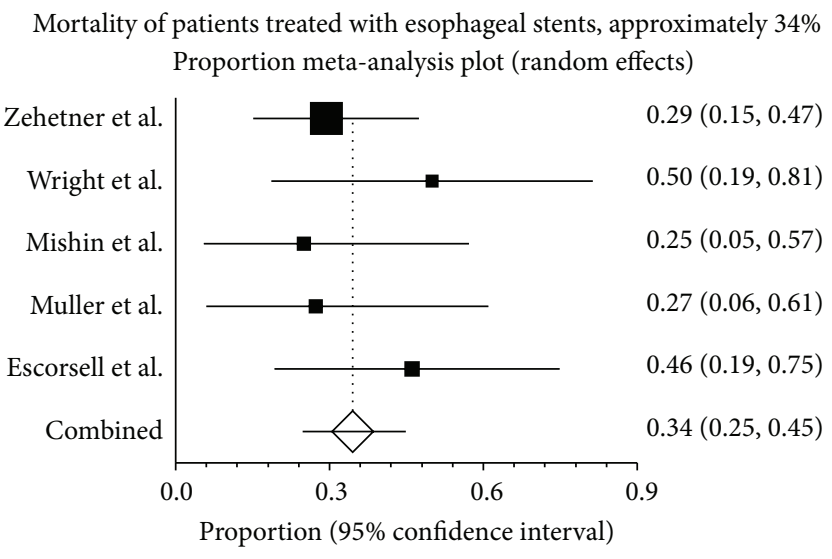

(a)

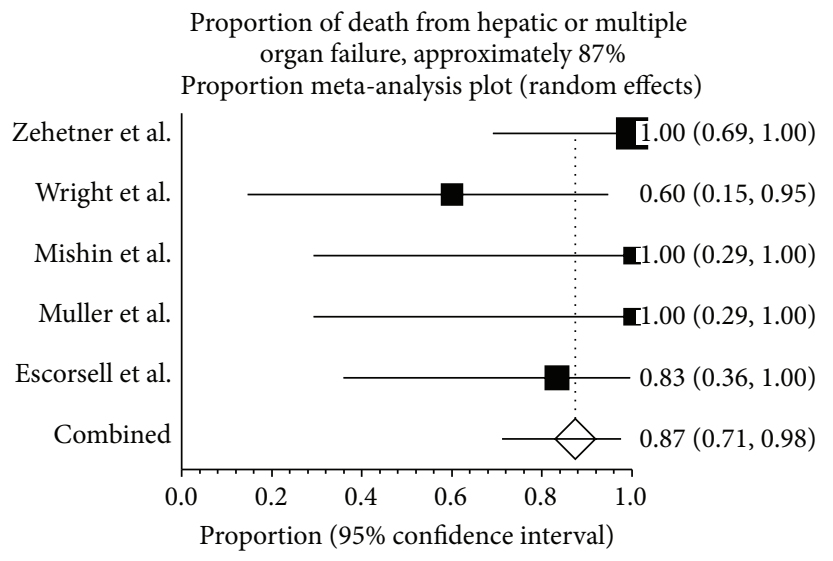

(b)

Proportion of death from uncontrolled bleeding, approximately $13 \%$ Proportion meta-analysis plot (random effects)

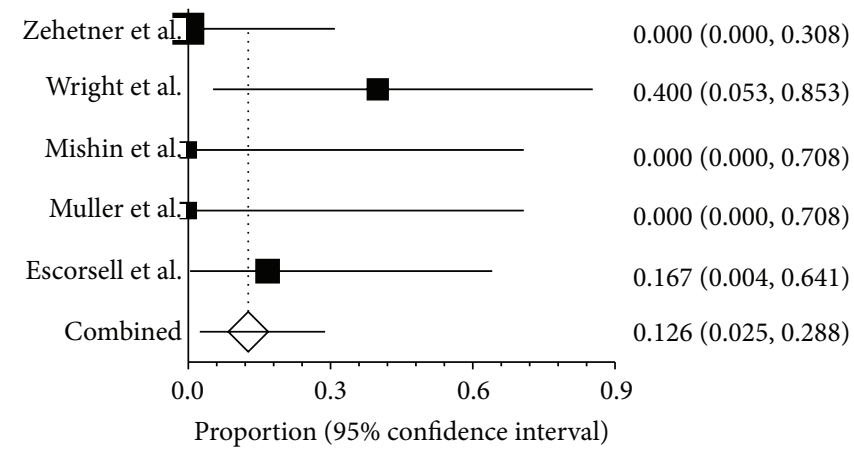

(c)

Figure 4: Mortality and causes of death of patients treated with esophageal stents. (a) Forest plot shows that about one-third [34.5\% (95\% CI: $24.8 \%-44.8 \%)$ ] of the 80 patients in the 5 studies died within 30 or $60 \mathrm{~d}$ of undergoing esophageal stents. There was no evidence of heterogeneity among studies $(P=0.60)$. (b) About nine-tenth [87.4\% (95\% CI: 71.2\%-97.5\%)] of deaths were due to hepatic or multiple organ failure in patients treated with esophageal stents. There was no evidence of heterogeneity among studies $(P=0.25)$. (c) Just over one-tenth [12.6\% (95\% CI: $2.5 \%-28.8 \%)]$ of deaths were contributed to uncontrolled bleeding. There was no evidence of heterogeneity among studies $(P=0.30)$. 
Incidence of stent migration, approximately $22 \%$ Proportion meta-analysis plot (random effects)

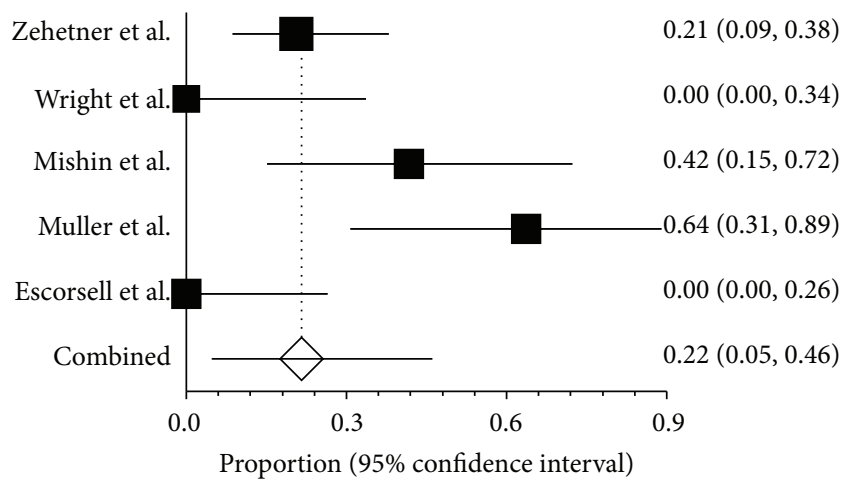

Figure 5: Stent migration after stent deployment. Stent migration was noted in about one-fifth [21.6\% (95\% CI: 4.7\%-46.1\%)] of the 80 patients in the 5 studies. There was, however, evidence of heterogeneity among the studies $(P=0.00)$.

rebleeding rate can be as high as 50\% [6]. TIPS is an alternative for refractory variceal bleeding and can achieve complete response in most cases [12]. Hepatic encephalopathy is the most common complication of TIPS [41]. Acute and acuteon-chronic liver failure are regarded as contraindications of TIPS [42, 43]. If these patients had undergone TIPS at the time of bleeding, the risk of early death after TIPS would have been very high, approaching $60 \%$ [44].

Esophageal stent is a nonsurgical approach that maintains the patency of esophagus in malignant or benign esophageal obstructions [45]. Esophageal stent is also used in dealing with esophageal perforation and fistula with a satisfactory outcome [18]. However, few studies explored its use in patients with refractory variceal bleeding. Consequently, the evidence on the effectiveness of esophageal stent in refractory variceal bleeding has been limited to a small number of study participants. Pooled results from 5 studies found a wide range of response and rebleeding rates, perhaps due to the lack of statistical power. In this study, we combined the data from these small studies, which allowed us to provide the best evidence on the effectiveness of esophageal stent in refractory variceal bleeding.

The stents used in the 5 studies are self-expandable covered esophageal metal stent (SX-ELLA-Danis, Czech Republic) that are specifically produced for controlling variceal bleeding [19]. The success rate of deploying stent in patients with acute variceal bleeding is $96.7 \%$. This proportion is well within the range for success rate of esophageal stenting in patients with malignant and benign esophageal strictures. Serious conditions, such as esophageal perforation [24] and acute bleeding, may not hinder the deployment of esophageal stents. Of course, the specific design of stents used in these studies may contribute to such a high success rate of procedures. However, in the earliest report of this procedure, five standard esophageal stents were also successfully inserted into expected positions with radiological guide [19]. Some reports indicated that the deployment of esophageal stent in patients with acute variceal bleeding is not a difficult procedure and specific designs of stents are more adequate in emergency room or intensive care unit without radiological facility [24]. In rare cases, the stents were inserted blindly without either X-ray or endoscopy [24]. However, Müller et al. pointed out that adequate training and exercise are necessary for successful deployment of esophageal stent in patients with acute variceal bleeding and blind stent implantation should not be advocated with concern of adverse events [26].

Refractory variceal bleeding is controlled after stent deployment in $93.9 \%$ of patients. Balloon tamponade is widely used in variceal bleeding which is adopted in $17.4 \%$ of patients with rebleeding [46]. Reportedly, $80 \%-90 \%$ of patients with refractory variceal bleeding treated with balloon tamponade achieved hemostasis [15]. This pooled analysis shows that in general the efficacy of esophageal stent on controlling bleeding seems not to be inferior to balloon tamponade. In the only RCT comparing esophageal stent and balloon tamponade, the primary composite endpoint (absence of digestive bleeding with absence of serious adverse events and survival at day 15) was achieved in $66 \%$ of the cases in the esophageal stent group but only in $20 \%$ in the balloon tamponade group [33]. According to this RCT, esophageal stent appears to be superior to balloon tamponade in treating refractory bleeding. In addition, esophageal stent allows patients to take food and drugs orally and undergo necessary diagnostic procedures including endoscopy. Esophageal stent is more comfortable than balloon tamponade for patients. The endotracheal intubation is not necessary for esophageal stenting, but balloon tamponade usually requires airway protection with intubation [38]. Balloon tamponade could be maintained for a maximum of $24-48 \mathrm{~h}$ to avoid esophageal or gastric pressure necrosis, but esophageal stents could remain in place for a longer period (range: 0-24 d). Thus, in hospitals where TIPS is not available, the longer retention time of stent is critical for safe transfer of patients to other medical centers. However, gastric varices will not be compressed by esophageal stent, so balloon tamponade still has a role in the management of gastric variceal bleeding.

With the use of banding ligation, vasoactive drugs, and antibiotics, the mortality of patients with variceal bleeding has been improved in recent years [47-50]. But initial failure to control bleeding or early rebleeding is usually associated with higher mortality [51]. The mortality rate increases to $80 \%$ if the initial treatment fails to stop acute bleeding [19]. In our study, the hemostasis rate was $94 \%$, but the overall mortality is still $34 \%$. These figures are higher than a general 6-week mortality rate of $15-20 \%$ using standard techniques [8]. This high mortality is attributed to the fact that all studies were conducted in high-risk patients with severe underlying liver diseases. The patients who are not responsive to standard therapy usually have advanced diseases and worse liver function. Child-Pugh class is the main predictor of outcome in patients with variceal bleeding [52]. Three of the 5 studies clearly recorded the Child-Pugh score, in which the proportion of patients with Child classes B and C is 77\%$100 \%$. Indeed, most of death cases $(87 \%)$ were from hepatic or multiple organ failure. The mortality rate of $34 \%$ in our study is lower than the results of previous studies conducted in 
patients with refractory variceal bleeding treated with other modalities [53].

The safety of esophageal stent in patients with variceal bleeding should be carefully assessed. The design of SXELLA-Danis stent with a protective pressure valve decreases the risk of perforation due to overinflated gastric balloon in esophagus [19]. In fact, two patients with concurrent variceal bleeding and esophagus perforation were successfully treated with this specifically designed stent [24]. Esophageal stenting followed by TIPS may be a reasonable choice for patients with variceal bleeding and perforation induced by balloon tamponade. There is no stent-related complication reported in the 5 studies, but an acute deterioration of pulmonary function was reported in a case report [23]. So the safety of this procedure should be further investigated in a larger number of patients. Stent migration occurred in about onefifth of patients but the dislocation, proximal or distal, is not associated with any major damage such as rebleeding, perforation, or obstruction. With close monitoring the stent migration was easily detected and repositioned with endoscopy.

Collectively, esophageal stent may be considered as an alternative to refractory variceal bleeding in future. However, our conclusions should be cautiously interpreted due to the following limitations. First, there is significant heterogeneity among studies in terms of length of follow-up and important endpoints such as complete response, incidence of rebleeding, and stent migration. Second, the quality of the studies is mediocre. Only one study was a small RCT. Third, the characteristics of the patients included in the studies are reported incompletely. Two studies did not report the Child-Pugh classification. Two studies did not report the presence of HCC. Two studies did not report the presence of previous bleeds. Fourth, the overall mortality in these studies is disappointingly high. Fifth, the stent has to be removed after a period of 1-2 weeks. Thus, additional strategies to lower portal pressure are warranted. The best combination of different modalities with esophageal stent needs further studies. In all of the 5 studies included in this analysis, esophageal stents serve as a temporary or bridging treatment followed by other therapies.

\section{Competing Interests}

The authors declare that there is no conflict of interests regarding the publication of this paper.

\section{References}

[1] G. Garcia-Tsao and J. Bosch, "Management of varices and variceal hemorrhage in cirrhosis," The New England Journal of Medicine, vol. 362, no. 9, pp. 778-832, 2010.

[2] G. D’Amico and R. de Franchis, "Upper digestive bleeding in cirrhosis. Post-therapeutic outcome and prognostic indicators," Hepatology, vol. 38, no. 3, pp. 599-612, 2003.

[3] B. Fortune and G. Garcia-Tsao, "Current management strategies for acute esophageal variceal hemorrhage," Current Hepatitis Reports, vol. 13, no. 1, pp. 35-42, 2014.
[4] R. De Franchis and M. Primignani, "Natural history of portal hypertension in patients with cirrhosis," Clinics in Liver Disease, vol. 5, no. 3, pp. 645-663, 2001.

[5] C. Merkel, M. Bolognesi, S. Bellon et al., "Prognostic usefulness of hepatic vein catheterization in patients with cirrhosis and esophageal varices," Gastroenterology, vol. 102, no. 3, pp. 973979, 1992.

[6] J. Bosch, A. Berzigotti, J. C. Garcia-Pagan, and J. G. Abraldes, "The management of portal hypertension: rational basis, available treatments and future options," Journal of Hepatology, vol. 48, no. 1, pp. S68-S92, 2008.

[7] N. Carbonell, A. Pauwels, L. Serfaty, O. Fourdan, V. G. Lévy, and R. Poupon, "Improved survival after variceal bleeding in patients with cirrhosis over the past two decades," Hepatology, vol. 40, no. 3, pp. 652-659, 2004.

[8] K. Stokkeland, L. Brandt, A. Ekbom, and R. Hultcrantz, "Improved prognosis for patients hospitalized with esophageal varices in Sweden 1969-2002," Hepatology, vol. 43, no. 3, pp. 500-505, 2006.

[9] G.-H. Lo, K.-H. Lai, J.-S. Cheng, M.-H. Chen, and H.-T. Chiang, "A prospective, randomized trial of butyl cyanoacrylate injection versus band ligation in the management of bleeding gastric varices," Hepatology, vol. 33, no. 5, pp. 1060-1064, 2001.

[10] R. Baares, A. Albillos, D. Rincón et al., "Endoscopic treatment versus endoscopic plus pharmacologic treatment for acute variceal bleeding: a meta-analysis," Hepatology, vol. 35, no. 3, pp. 609-615, 2002.

[11] G.-H. Lo, K.-H. Lai, W.-W. Ng et al., "Injection sclerotherapy preceded by esophageal tamponade versus immediate sclerotherapy in arresting active variceal bleeding: a prospective randomized trial," Gastrointestinal Endoscopy, vol. 38, no. 4, pp. 421-424, 1992.

[12] G. Garcia-Tsao, A. J. Sanyal, N. D. Grace et al., "Prevention and management of gastroesophageal varices and variceal hemorrhage in cirrhosis," Hepatology (Baltimore, Md.), vol. 46, no. 3, pp. 922-938, 2007.

[13] J. C. García-Pagán, K. Caca, C. Bureau et al., "Early use of TIPS in patients with cirrhosis and variceal bleeding," The New England Journal of Medicine, vol. 362, no. 25, pp. 2370-2379, 2010.

[14] À. Escorsell and J. Bosch, "Self-expandable metal stents in the treatment of acute esophageal variceal bleeding," Gastroenterology Research and Practice, vol. 2011, Article ID 910986, 6 pages, 2011.

[15] A. Avgerinos and A. Armonis, "Balloon tamponade technique and efficacy in variceal haemorrhage," Scandinavian Journal of Gastroenterology, vol. 29, no. 207, pp. 11-16, 1994.

[16] M. D’Amico, A. Berzigotti, and J. C. Garcia-Pagan, "Refractory acute variceal bleeding: what to do next?" Clinics in Liver Disease, vol. 14, no. 2, pp. 297-305, 2010.

[17] G. D’Amico, L. Pagliaro, and J. Bosch, "The treatment of portal hypertension: a meta-analytic review," Hepatology, vol. 22, no. 1, pp. 332-354, 1995.

[18] P. Sharma and R. Kozarek, "Role of esophageal stents in benign and malignant diseases," The American Journal of Gastroenterology, vol. 105, no. 2, pp. 258-273, 2010.

[19] R. Hubmann, G. Bodlaj, M. Czompo et al., "The use of self-expanding metal stents to treat acute esophageal variceal bleeding," Endoscopy, vol. 38, no. 9, pp. 896-901, 2006.

[20] J. Zehetner, A. Shamiyeh, W. Wayand, and R. Hubmann, "Results of a new method to stop acute bleeding from 
esophageal varices: implantation of a self-expanding stent," Surgical Endoscopy and Other Interventional Techniques, vol. 22, no. 10, pp. 2149-2152, 2008.

[21] A. Dechene, A. El Fouly, E. J. De Dechêne, T. Zoepf, and G. Gerken, "S1510: the bleeding stopped-what now? Selfexpanding metal stents (SEMS) in refractory variceal bleeding and as part of combination therapy," Gastrointestinal Endoscopy, vol. 71, no. 5, p. AB181, 2010.

[22] W.-R. Matull, T. J. S. Cross, D. Yu, M. C. Winslet, and J. O'Beirne, "A removable covered self-expanding metal stent for the management of Sengstaken-Blakemore tube-induced esophageal tear and variceal hemorrhage," Gastrointestinal Endoscopy, vol. 68, no. 4, pp. 767-768, 2008.

[23] A. Dechene, M. Adamzik, G. Gerken, and A. Canbay, "Acute bronchial obstruction following esophageal stent implantation for variceal bleeding," Endoscopy, vol. 41, supplement 2, pp. E146-E147, 2009.

[24] G. Wright, H. Lewis, B. Hogan, A. Burroughs, D. Patch, and J. O'Beirne, "A self-expanding metal stent for complicated variceal hemorrhage: experience at a single center," Gastrointestinal Endoscopy, vol. 71, no. 1, pp. 71-78, 2010.

[25] I. Mishin, G. Ghidirim, A. Dolghii, G. Bunic, and G. Zastavnitsky, "Implantation of self-expanding metal stent in the treatment of severe bleeding from esophageal ulcer after endoscopic band ligation," Diseases of the Esophagus, vol. 23, no. 7, pp. E35E38, 2010.

[26] M. Müller, T. Seufferlein, L. Perkhofer, M. Wagner, and A. Kleger, "Self-expandable metal stents for persisting esophageal variceal bleeding after band ligation or injection-therapy: a retrospective study," PLOS ONE, vol. 10, no. 6, Article ID e0126525, 2015.

[27] M. S. Zakaria, I. M. Hamza, M. A. Mohey, and R. G. Hubamnn, "The first Egyptian experience using new self-expandable metal stents in acute esophageal variceal bleeding: Pilot study," Saudi Journal of Gastroenterology, vol. 19, no. 4, pp. 177-181, 2013.

[28] F. C. Fierz, W. Kistler, V. Stenz, and C. Gubler, "Treatment of esophageal variceal hemorrhage with self-expanding metal stents as a rescue maneuver in a swiss multicentric cohort," Case Reports in Gastroenterology, vol. 7, no. 1, pp. 97-105, 2013.

[29] I. L. Holster, E. J. Kuipers, H. R. Van Buuren, M. C. W. Spaander, and E. T. T. L. Tjwa, "Self-expandable metal stents as definitive treatment for esophageal variceal bleeding," Endoscopy, vol. 45, no. 6, pp. 485-488, 2013.

[30] A. Dechêne, A. H. El Fouly, L. P. Bechmann et al., "Acute management of refractory variceal bleeding in liver cirrhosis by self-expanding metal stents," Digestion, vol. 85, no. 3, pp. 185191, 2012.

[31] B. S. Ravindra, N. Ganjoo, E. B. Maanthagonda, and K. Shinde, "Esophageal variceal stent for uncontrolled post endoscopic variceal ligation ulcer bleed," Indian Journal of Gastroenterology, vol. 32, supplement 1, no. 1, p. A12, 2013.

[32] I. Mishin, G. Zastavnitsky, G. Ghidirim, and G. Bunic, "Selfexpanding metal stents: a new hemostasis method for bleeding esophageal varices," Hepatology International, vol. 7, supplement 1, article S540, 2013.

[33] À. Escorsell, O. Pavel, A. Cárdenas et al., "Esophageal balloon tamponade versus esophageal stent in controlling acute refractory variceal bleeding: a multicenter randomized, controlled trial," Hepatology, vol. 63, no. 6, pp. 1957-1967, 2016.

[34] B. Feneyrou, J. Hanana, J. P. Daures, and J. B. Prioton, "Initial control of bleeding from esophageal varices with the
Sengstaken-Blakemore tube. Experience in 82 patients," The American Journal of Surgery, vol. 155, no. 3, pp. 509-511, 1988.

[35] R. De Franchis, "Revising consensus in portal hypertension: report of the Baveno v consensus workshop on methodology of diagnosis and therapy in portal hypertension," Journal of Hepatology, vol. 53, no. 4, pp. 762-768, 2010.

[36] C.-F. Chong, "Esophageal rupture due to Sengstaken-Blakemore tube misplacement," World Journal of Gastroenterology, vol. 11, no. 41, pp. 6563-6565, 2005.

[37] A. Avgerinos, C. Klonis, G. Rekoumis, P. Gouma, N. Papadimitriou, and S. Raptis, "A prospective randomized trial comparing somatostatin, balloon tamponade and the combination of both methods in the management of acute variceal haemorrhage," Journal of Hepatology, vol. 13, no. 1, pp. 78-83, 1991.

[38] J. Panés, J. Terés, J. Bosch, and J. Rodés, "Efficacy of balloon tamponade in treatment of bleeding gastric and esophageal varices. Results in 151 consecutive episodes," Digestive Diseases and Sciences, vol. 33, no. 4, pp. 454-459, 1988.

[39] R. de Franchis, G. M. Eisen, L. Laine et al., "Esophageal capsule endoscopy for screening and surveillance of esophageal varices in patients with portal hypertension," Hepatology, vol. 47, no. 5, pp. 1595-1603, 2008.

[40] T. C. Collyer, S. E. T. Dawson, and D. Earl, "Acute upper airway obstruction due to displacement of a Sengstaken-Blakemore tube," European Journal of Anaesthesiology, vol. 25, no. 4, pp. 341-342, 2008.

[41] M. Rössle, “TIPS: 25 years later," Journal of Hepatology, vol. 59, no. 5, pp. 1081-1093, 2013.

[42] Z. Hassoun, M. Deschênes, M. Lafortune et al., "Relationship between pre-TIPS liver perfusion by the portal vein and the incidence of post-TIPS chronic hepatic encephalopathy," The American Journal of Gastroenterology, vol. 96, no. 4, pp. 12051209, 2001.

[43] T. D. Boyer and Z. J. Haskal, "The role of transjugular intrahepatic portosystemic shunt in the management of portal hypertension," Hepatology, vol. 41, no. 2, pp. 386-400, 2005.

[44] J. E. Lopera, "Role of emergency transjugular intrahepatic portosystemic shunts," Seminars in Interventional Radiology, vol. 22, no. 4, pp. 253-265, 2005.

[45] A. J. Dormann, S. Meisner, N. Verin, and A. Wenk Lang, "Self-expanding metal stents for gastroduodenal malignancies: systematic review of their clinical effectiveness," Endoscopy, vol. 36, no. 6, pp. 543-550, 2004.

[46] D. Cook and L. Laine, "Indications, technique, and complications of balloon tamponade for variceal gastrointestinal bleeding," Journal of Intensive Care Medicine, vol. 7, no. 4, pp. 212-218, 1992.

[47] A. E. S. Gimson, J. K. Ramage, M. Z. Panos et al., "Randomised trial of variceal banding ligation versus injection sclerotherapy for bleeding oesophageal varices," The Lancet, vol. 342, no. 8868, pp. 391-394, 1993.

[48] G. Ioannou, J. Doust, and D. C. Rockey, "Terlipressin for acute esophageal variceal hemorrhage," Cochrane Database of Systematic Reviews, no. 1, Article ID CD002147, 2001.

[49] M.-C. Hou, H.-C. Lin, T.-T. Liu et al., "Antibiotic prophylaxis after endoscopic therapy prevents rebleeding in acute variceal hemorrhage: a randomized trial," Hepatology, vol. 39, no. 3, pp. 746-753, 2004.

[50] A. K. Burroughs, C. K. Triantos, J. O’Beirne, and D. Patch, "Predictors of early rebleeding and mortality after acute variceal hemorrhage in patients with cirrhosis," Nature Clinical Practice Gastroenterology and Hepatology, vol. 6, no. 2, pp. 72-73, 2009. 
[51] Z. Ben-Ari, F. Cardin, A. P. McCormick, G. Wannamethee, and A. K. Burroughs, "A predictive model for failure to control bleeding during acute variceal haemorrhage," Journal of Hepatology, vol. 31, no. 3, pp. 443-450, 1999.

[52] L. Amitrano, M. A. Guardascione, F. Manguso et al., "The effectiveness of current acute variceal bleed treatments in unselected cirrhotic patients: refining short-term prognosis and risk factors," The American Journal of Gastroenterology, vol. 107, no. 12, pp. 1872-1878, 2012.

[53] J. C. Garcia-Pagán and J. Bosch, "Endoscopic band ligation in the treatment of portal hypertension," Nature Clinical Practice Gastroenterology and Hepatology, vol. 2, no. 11, pp. 526-535, 2005. 


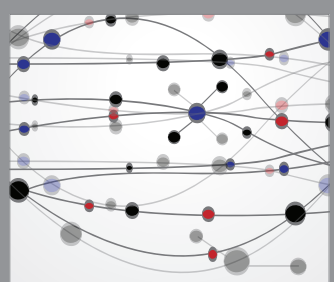

The Scientific World Journal
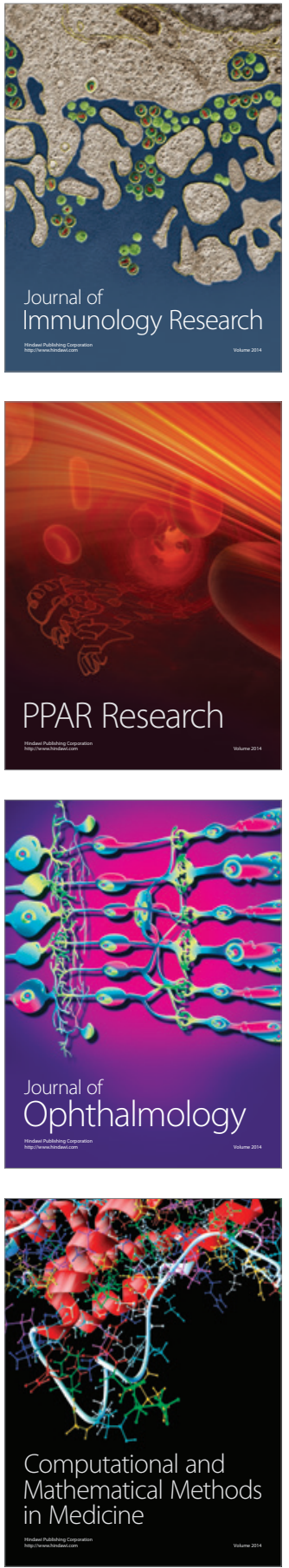

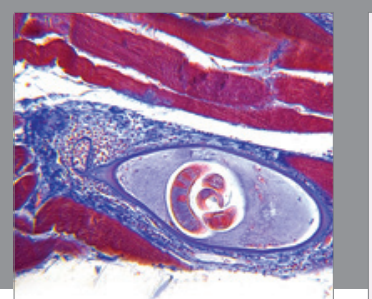

Gastroenterology Research and Practice

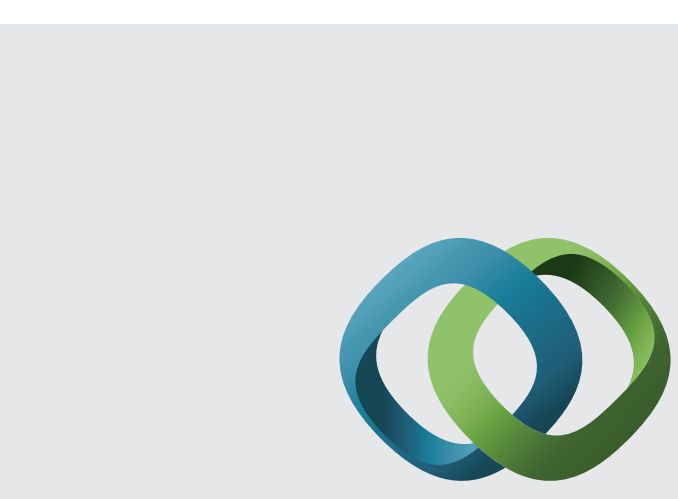

\section{Hindawi}

Submit your manuscripts at

http://www.hindawi.com
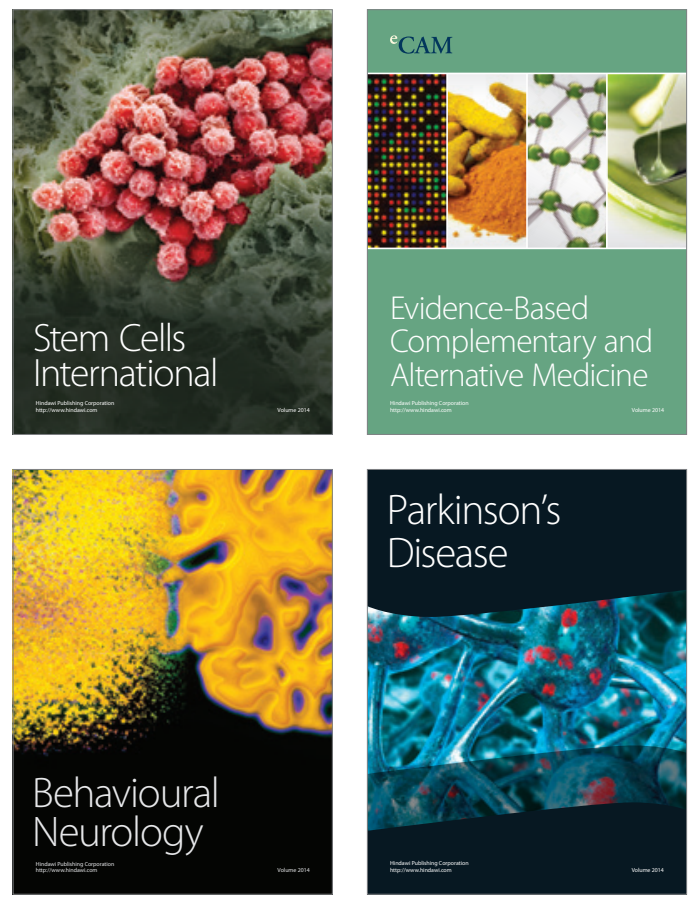
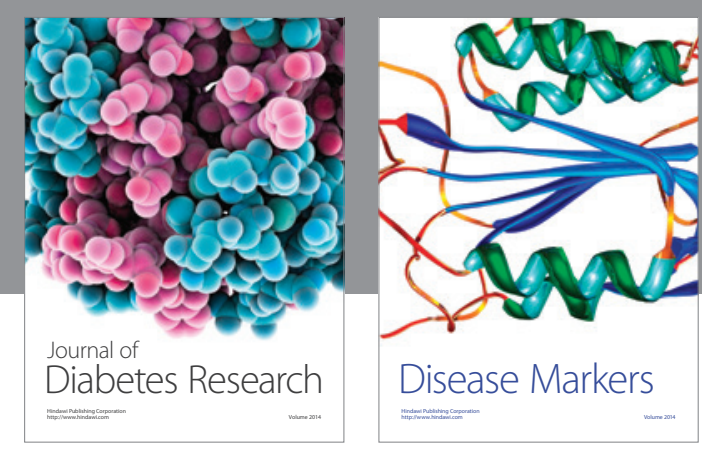

Disease Markers
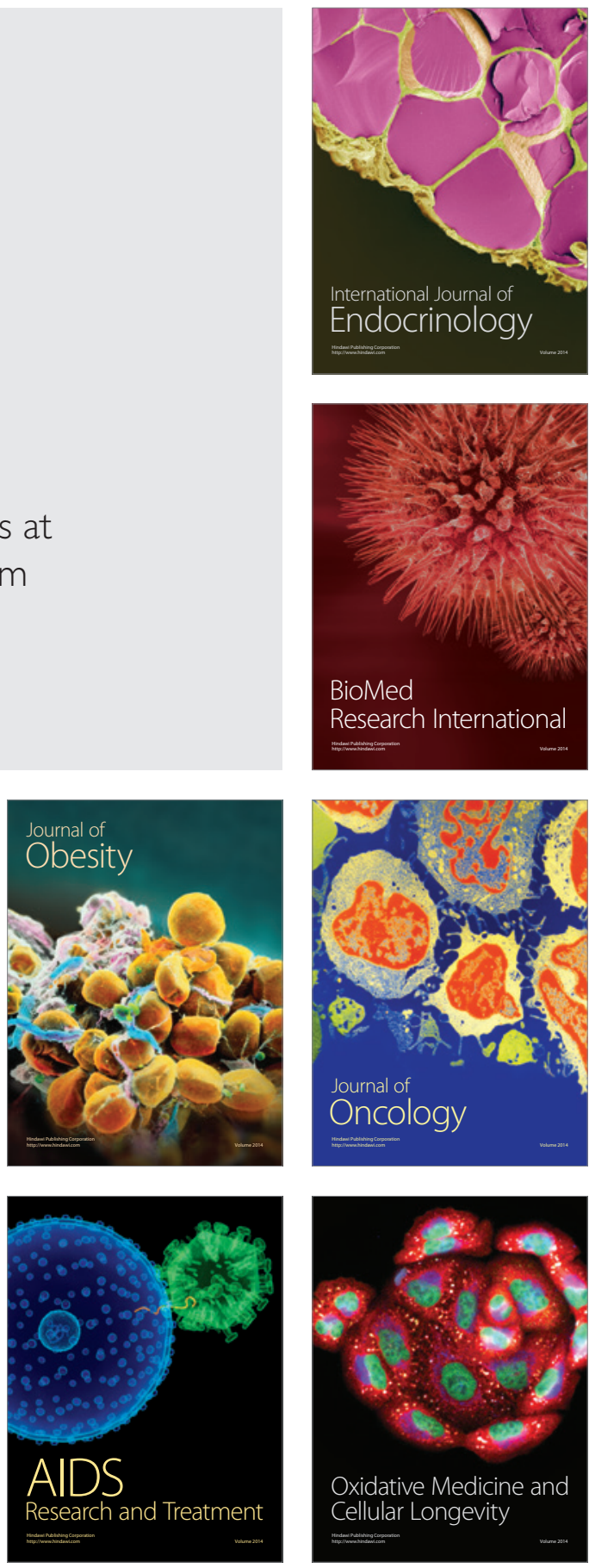